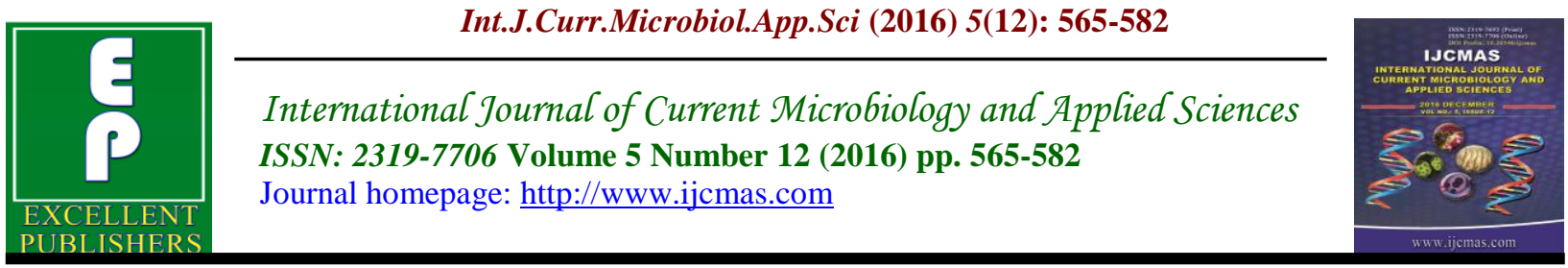

Original Research Article

http://dx.doi.org/10.20546/ijcmas.2016.512.062

\title{
Phylogenetic Analysis of Endophytic Bacteria from Nakshtra Trees
}

\author{
Priya Dnyandeo Kakade ${ }^{1 *}$ and Sushma Ravindra Chaphalkar ${ }^{2}$
}

Vidya Pratishthan's School of Biotechnology, Vidyanagari, Baramati, Pune-413133, India

*Corresponding author:

\section{Keywords}

Endophytic bacteria, 16 s rDNA technique, Phylogenetic analysis, Antimicrobial

Peptides,

Biocontrol agent.

\section{Article Info}

Accepted:

18 November 2016

Available Online:

10 December 2016

\section{A B S T R A C T}

Endophytic bacteria were inhabitant microflora of plant part. This association was presumably long term and they were mostly commensalisms. The microorganisms which were living within the plant cell without causing any harmful effect on the plant tissue. These bacteria were secrets the plant growth promoting hormones like indole acetic acid which helps for growth of plant. In the present study the endophytic bacteria was isolated from medicinal plants of nakshtra udyan and screened out for the different characteristics of these microorganisms. The isolates were identified by 16s $r$ DNA technique and the phylogenetic analysis was done for the 96 isolates from Nakshtra Udyan plant. The isolates were grouped into 23 different types of group of species and subspecies of microorganisms in which Bacillus species are more predominant than Pseudomonas sp, Enterobacter $s p$, Alcaligene spp, Arthrobacter sp, Micrococcus sp, Klebsiella pneumoniae and kocuria sp. These bacteria were important for different properties like antimicrobial peptides, plant growth promoting hormones and used as biocontrol agent.

\section{Introduction}

A niche environment previously untapped has been explored for study of bacterial population within leaf portions of huge medicinal trees. This study has yielded into isolation of a few peculiarly characterized as endophytic bacteria hosting an unique culture collection at VSBT. This culture collection of endophytic bacteria has yielded into phylogenetic patterns.

They are known to secrete different types of secondary metabolites like antimicrobial peptides, growth promoting hormones and bioactive compounds which are used as biocontrol agent, in pharmacology used as antimicrobial drugs preparation, in food industry and agriculture (Dhanya et al., 2013
Susheel kumar et al., 2013). These endophytic bacteria play an important role for improvement in growth and health of plant and it has several mechanisms for it (Taghavi et al., 2009). Endophytes may directly produce chemical defense in plants through the production of secondary compounds which inhibit insects and pathogenic organisms. The in vitro secretion of substances by endophytes that limit the growth of other microbial species, including pathogens (Vania Specian et al., 2012). The many of endophytes re poorly investigated group of microorganisms that produces secondary metabolites which is used in modern medicine and about $40 \%$ of the prescription of drugs are based on them 
(Shukla et al., 2014). The endophytic bacteria produces Indole Acetic acid ( IAA) (Pedraza et al., 2004), cytokinin (Ergun et al., 2002) and Gibberellic acid (GA) (Kharwar et al., 2008) which are required for the plant growth. In the 70's, endophytes were initially considered neutral, neither causing benefits nor showing detrimental influence on plants, but from the results of more recent studies it has been possible to show that in many cases, they have an important role in host protection against pathogens. Several studies have now shown that the interaction between plants and some endophytic bacteria is associated with beneficial effects such as plant growth promotion and biocontrol potential against plant pathogens (Lalande et al., 1989; Bashan et al., 1990; Chen et al., 1995; Hallmann et al., 1997).

\section{Materials and Methods}

\section{Selection of medicinal plants and Explants collection}

For the isolation of bacteria the explants were collected from different parts of medicinal plants from Nakshtra Udyan which located at Vidya Pratishthan's school of biotechnology. The cultivation of these plants was strictly maintained in organic package and the age of plants was fourteen years.

\section{Pretreatment and surface sterilization of explants}

The explants used for the isolation were leaves, stem and root of the plants. The collected explants was brought to the laboratory and washed under running tap water. After this these were thoroughly washed with distilled water. Surface sterilization protocol was standardized which contained surface sterilizing agents like $1 \%$ phenolic compounds containing solution for $5 \mathrm{~min}$ followed by $0.1 \%$ sodium hypochlorite treatment for $5 \mathrm{~min}$. Afterwards the explants was washed with sterile distilled water.

\section{Isolation of endophytic bacteria}

The samples were aseptically ground in a motor and pestle in potassium dihydrogen phosphate buffer ( $\mathrm{pH}$ 6.8) and inoculated into sterile nutrient broth medium with negative and positive control i.e. sterilized medium without inoculation of explants and uncrushed surface sterilized explants. The broth was incubated for $24 \mathrm{hrs}$ at $30^{\circ} \mathrm{C}$ on rotary shaker incubator at $120 \mathrm{rpm}$. The grown culture was plated onto sterile nutrient agar plates. The plates were incubated at $37 \mathrm{C}$ for $24 \mathrm{hrs}$. The isolated bacteria were plated onto selective medium after their morphological, biochemical and molecular identification and preserve it by lyophilization.

\section{Biochemical characterization of isolates}

The standard tests for the characterization were done according to the Beregey's Mannual of Determinative Bacteriology. The isolates were characterized for colony characterization which includes size, shape, color, consistency, opacity, Gram's nature, Capsule staining and presence of endospores. The biochemical characterization was done for IMViC test, starch hydrolysis, gelatin liquefaction, and different sources of carbon utilization, Oxidase and catalase tests by standard methods.

\section{Molecular Characterization of isolates}

The isolated strains were identified by using 16 s r DNA techniques. The genomic DNA of endophytic bacteria was isolated by using 
CTAB method. The amplification of template DNA was done by using universal primes, R1 forward (5'AGTTTGAT CCTGGCTCAG 3') and R2 reverse (5' GGACTACCAGGGTATCTAAT3'). The 50 ul PCR reaction contains $\mathrm{Mgcl}_{2}$ $(0.45 \mathrm{mM})$, dNTPs $(0.2 \mathrm{mM})$, forward primer $(10 \mathrm{pmol})$, reverse primer $(10 \mathrm{pmol})$, Taq polymerase $(0.5 \mathrm{U})$ having $10 \mathrm{X}$ assay buffer $(1 \mathrm{X})$, genomic DNA(1 ug/ul) and sterile MilliQ water is used. Amplification of DNA was done in automated thermocycle machine provided by applied biosystem and product was checked on $1 \%$ agarose gel. The gel was eluted by using SIGMA gel elution kit.

The sequencing reaction was carried out in 3130 genetic analyzer at VSBT. The BLAST of the sequences was done for sequences of bacteria to NCBI GeneBank.

\section{Phylogenetic tree analysis}

The phylogenetic tree was constructing by using MEGA software. The neighborjoining method was used to construct the phylogenetic tree. The bootstrap resampling test with 100 replications was also applied.

\section{Results and Discussion}

These observation form one of the most significant and comparable account of endophytic bacteria in tropical tree species (Table.1). This high through put isolation followed by classification and identification of endophytic bacteria using biochemical and $16 \mathrm{~s}$ rDNA techniques has culminated into reliable unique culture collection center at our center. Biochemical preferences of these bacteria has shown acetate as preferred carbon source only to put forth a heavy duty of acetate scavenging function in tree species in longest longitude. The phylogeny study specifies an almost ubiquitous presence of Bacillus sp. Among 96 endophytic bacterial isolates 34 were from the same group i.e. Bacillus sp. (Table.2).

Table.1 Plants used for isolation of endophytic bacteria.

\begin{tabular}{|l|l|l|l|l|}
\hline Sr. No. & $\begin{array}{l}\text { Name of the } \\
\text { plant }\end{array}$ & Botanical Name & Plant family & Image of plant \\
\hline 1 & Adulsa & Adhatoda vasica & Acanthaceae \\
\hline 2 & Arjun & $\begin{array}{l}\text { Terminalia } \\
\text { arjuna }\end{array}$ & Combretaceae & \\
\hline
\end{tabular}


Int.J.Curr.Microbiol.App.Sci (2016) 5(12): $565-582$

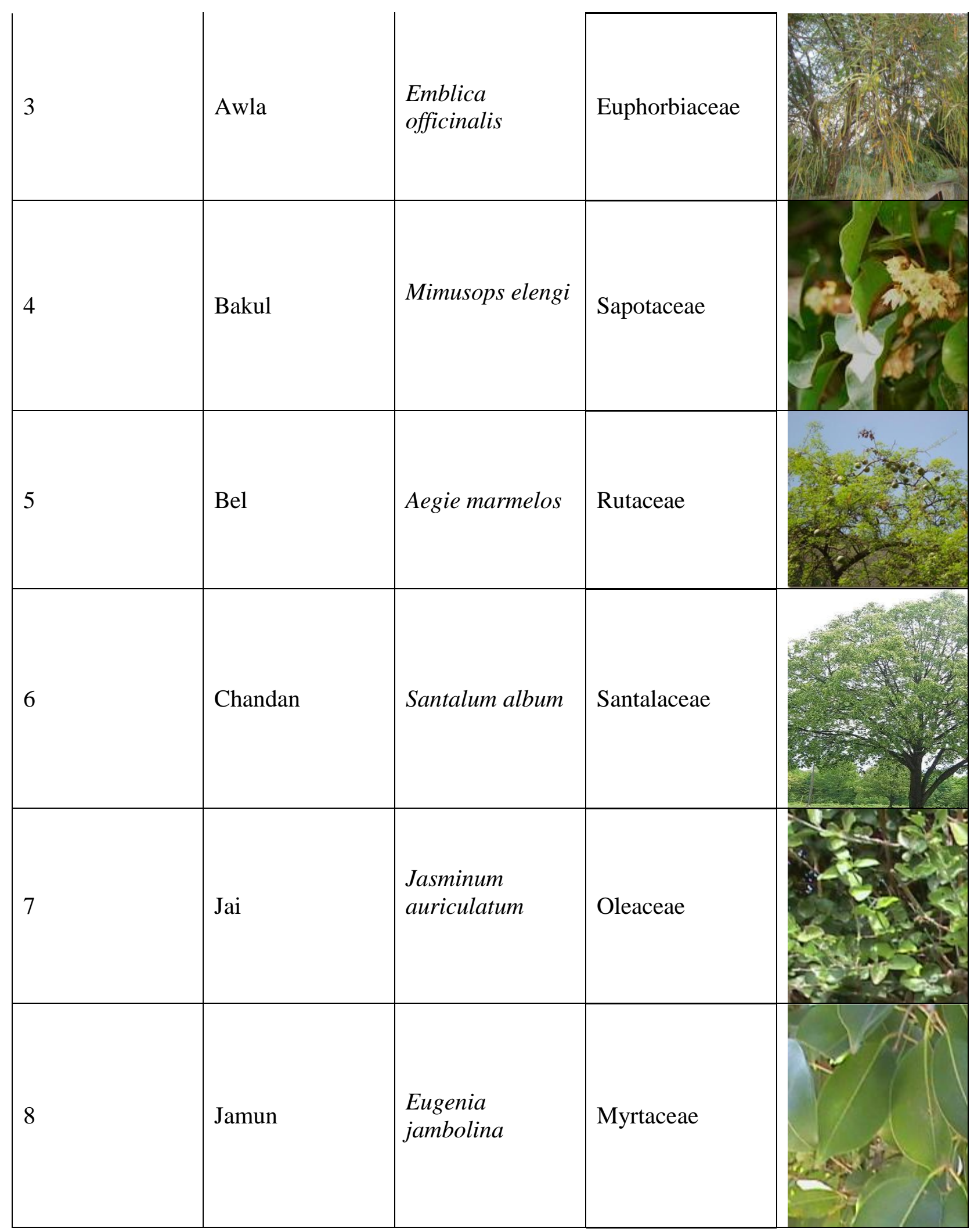


Int.J.Curr.Microbiol.App.Sci (2016) 5(12): $565-582$

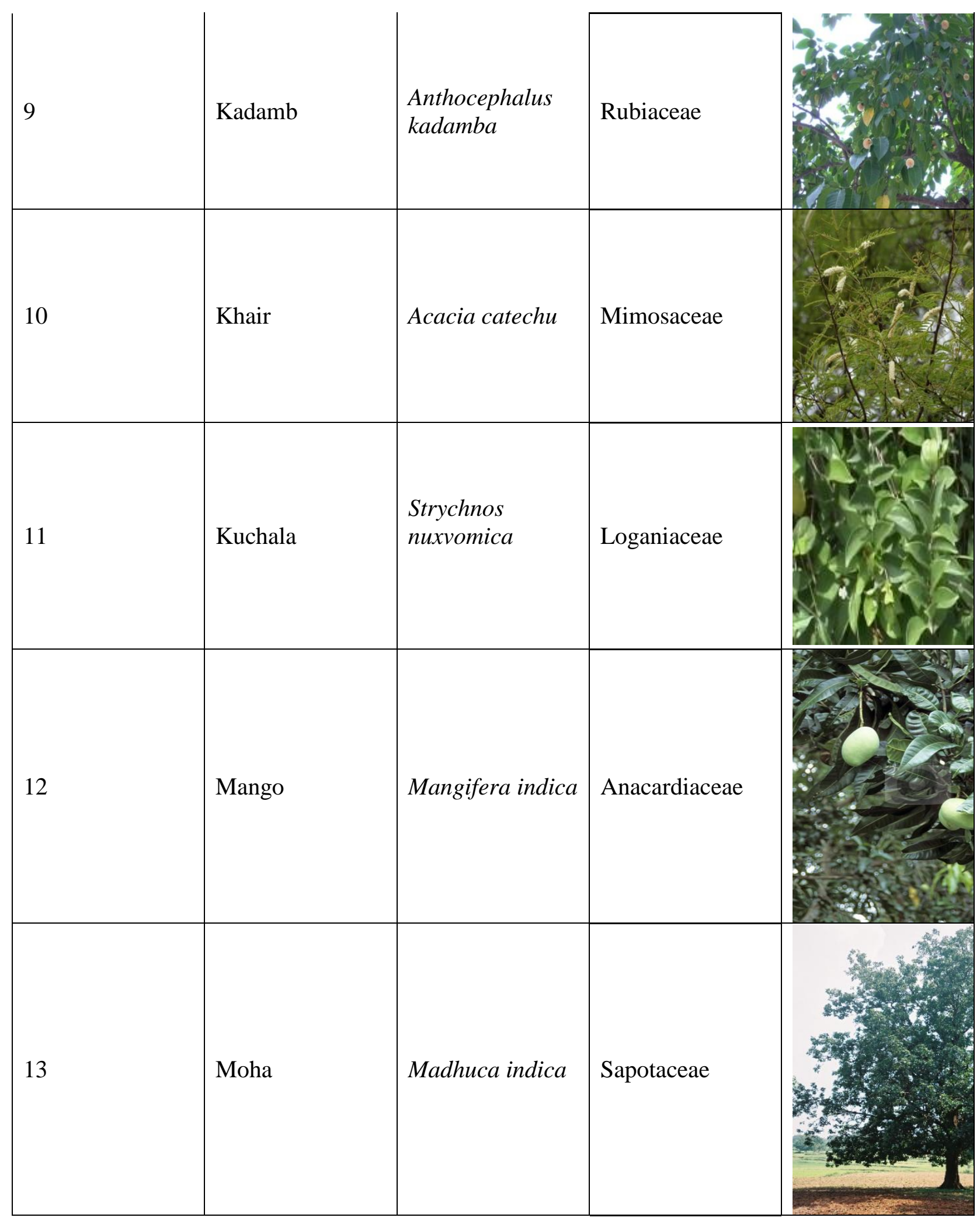


Int.J.Curr.Microbiol.App.Sci (2016) 5(12): $565-582$

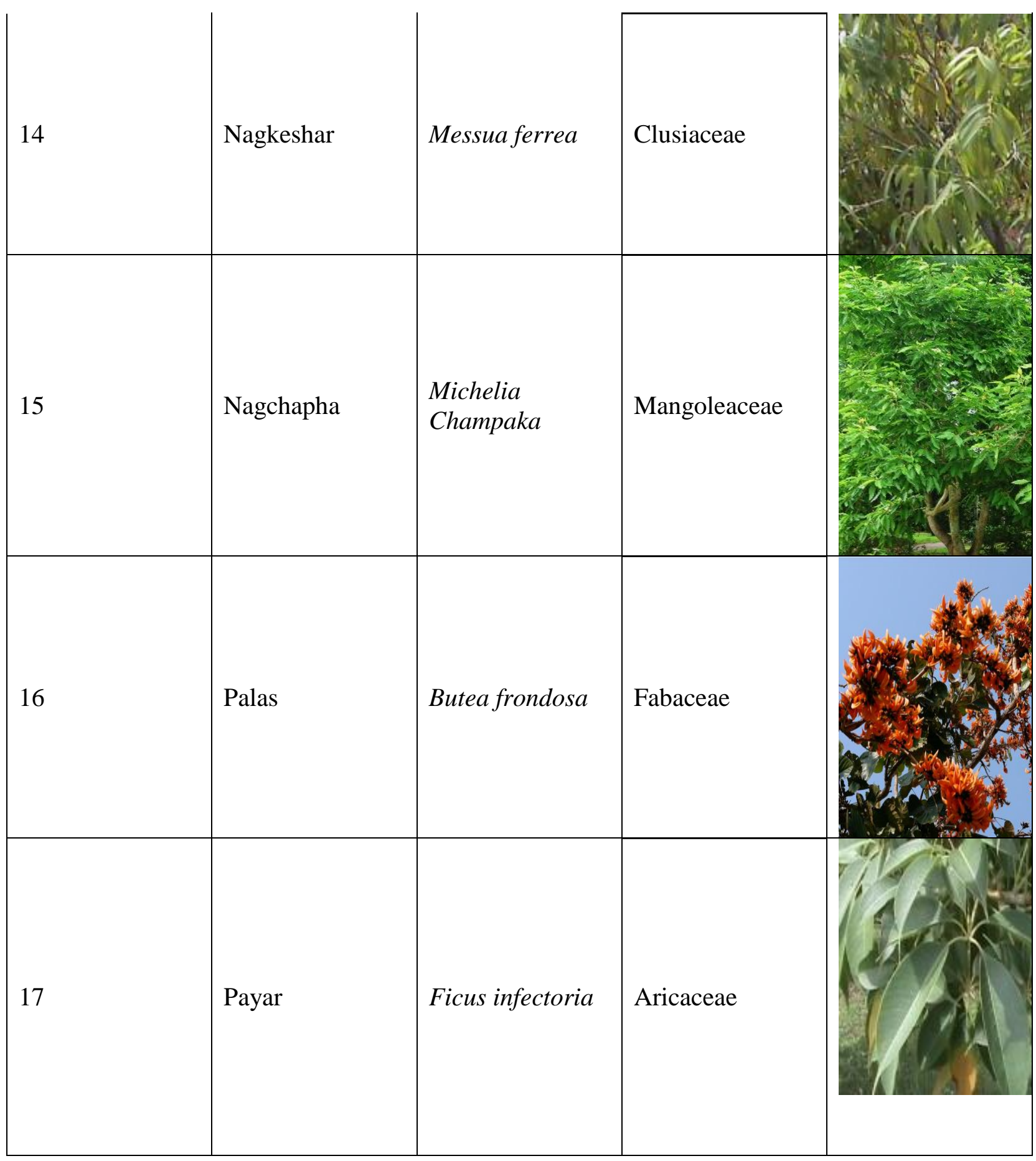


Int.J.Curr.Microbiol.App.Sci (2016) 5(12): $565-582$

\begin{tabular}{|c|c|c|c|c|}
\hline 18 & Phanas & $\begin{array}{l}\text { Artocarpus } \\
\text { iniegrifolia }\end{array}$ & Moraceae & 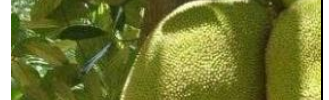 \\
\hline 19 & Pimpal & Ficus religiosa & Articaceae & \\
\hline 20 & Raal & Veteria indica & Dipterocarpaceae & \\
\hline 21 & Rui & $\begin{array}{l}\text { Calotropis } \\
\text { gigantea }\end{array}$ & Asclepiadiaceae & \\
\hline 22 & Savar & $\begin{array}{l}\text { Salmalia } \\
\text { malabarica }\end{array}$ & Malvaceae & $\frac{1}{25}$ \\
\hline
\end{tabular}


Int.J.Curr.Microbiol.App.Sci (2016) 5(12): $565-582$

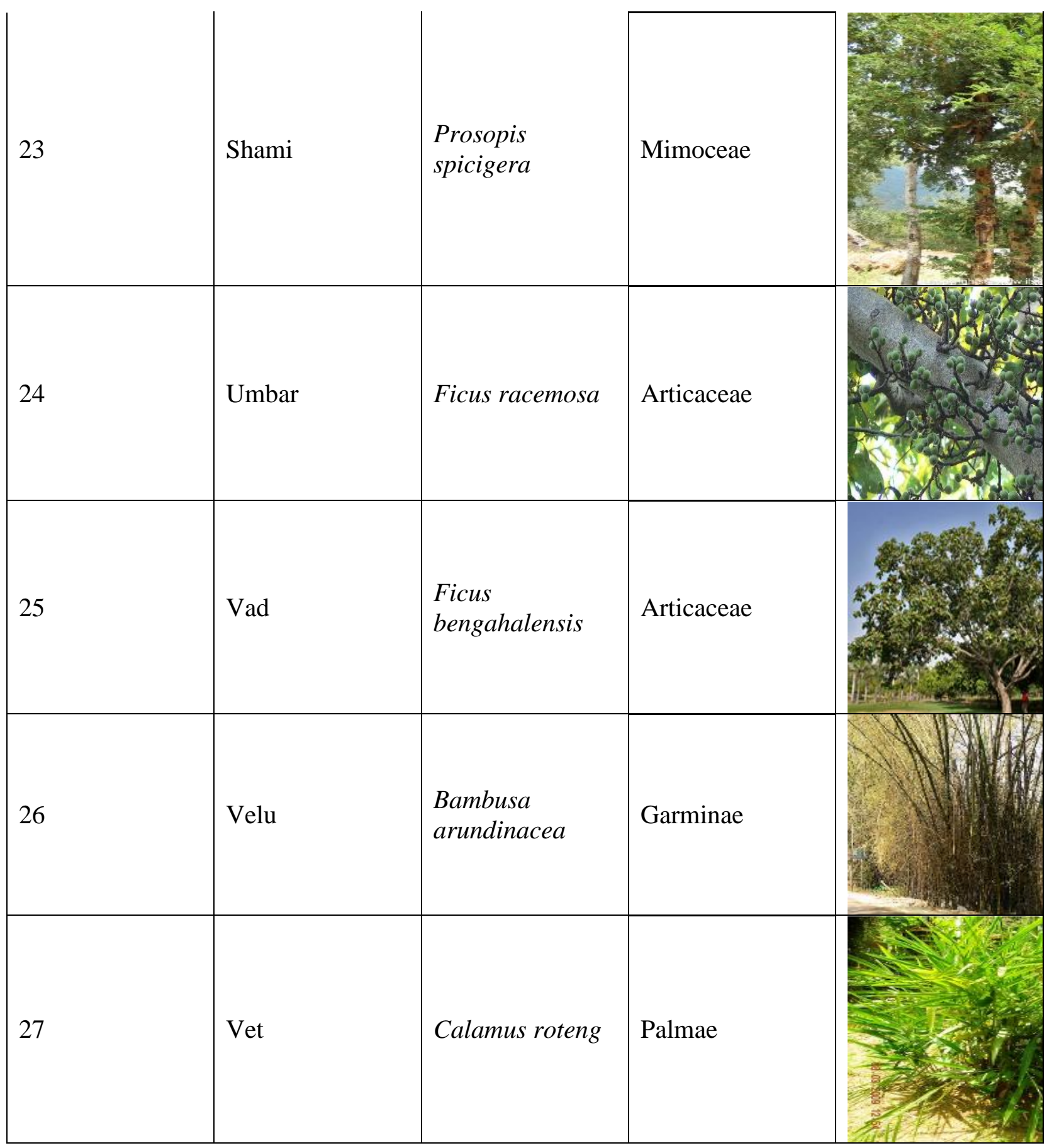


Table.2 Classification of endophytic bacteria on the basis of identification:

\begin{tabular}{|c|c|c|c|c|c|}
\hline $\begin{array}{l}\text { Name of } \\
\text { isolated } \\
\text { bacterial } \\
\text { species }\end{array}$ & $\begin{array}{l}\text { Total number } \\
\text { of new strains }\end{array}$ & Code of isolate & Endo Code & $\begin{array}{l}\text { Name of plant } \\
\text { and plant part } \\
\text { used }\end{array}$ & $\begin{array}{l}\text { Sequence } \\
\text { length }\end{array}$ \\
\hline \multirow[t]{2}{*}{ Bacillus subtilis } & \multirow[t]{2}{*}{02} & VCC.16.SB & Endo_25 & $\begin{array}{l}\text { Messua ferrea } \\
\text { Stem }\end{array}$ & 776 \\
\hline & & VCC.5.S & Endo_1 & $\begin{array}{l}\text { Acacia catechu } \\
\text { Stem }\end{array}$ & 776 \\
\hline \multirow[t]{3}{*}{$\begin{array}{l}\text { Bacillus } \\
\text { megaterium }\end{array}$} & \multirow[t]{3}{*}{03} & VCC.4.RA, & Endo_7 & $\begin{array}{l}\text { Eugenia } \\
\text { jambolina Stem, }\end{array}$ & 789 \\
\hline & & VCC.22.LD & Endo_35 & $\begin{array}{l}\text { Anthocephalus } \\
\text { kadamba leaf }\end{array}$ & 799 \\
\hline & & VCC.4.SB & Endo_21 & $\begin{array}{l}\text { Eugenia } \\
\text { jambolina Stem }\end{array}$ & 793 \\
\hline \multirow[t]{2}{*}{$\begin{array}{l}\text { Bacillus } \\
\text { lichniformis }\end{array}$} & \multirow[t]{2}{*}{02} & VCC.8.SA & Endo_12 & $\begin{array}{ll}\text { Ficus } & \text { religiosa } \\
\text { Stem } & \\
\end{array}$ & 701 \\
\hline & & VCC.15.LE & Endo_82 & $\begin{array}{l}\text { Terminalia arjuna } \\
\text { leaf }\end{array}$ & 754 \\
\hline $\begin{array}{l}\text { Bacillus } \\
\text { axarequiensis }\end{array}$ & 01 & VCC.22.LC & Endo_81 & $\begin{array}{l}\text { Anthocephalus } \\
\text { kadamba leaf }\end{array}$ & 773 \\
\hline \multirow[t]{2}{*}{$\begin{array}{l}\text { Bacillus } \\
\text { safensis }\end{array}$} & \multirow[t]{2}{*}{02} & VCC.7.LC & Endo_71 & $\begin{array}{l}\text { Bambusa } \\
\text { arundinacea leaf }\end{array}$ & 751 \\
\hline & & VCC.21.L & Endo_83 & $\begin{array}{l}\text { Prosopis } \\
\text { spicigera leaf }\end{array}$ & 766 \\
\hline Bacillus firmus & 01 & VCC.26.LB & Endo_86 & $\begin{array}{l}\text { Calamus roteng } \\
\text { leaf }\end{array}$ & 768 \\
\hline $\begin{array}{l}\text { Bacillus } \\
\text { pumilus }\end{array}$ & 01 & VCC.22.LG & Endo_50 & $\begin{array}{l}\text { Anthocephalus } \\
\text { kadamba leaf }\end{array}$ & 700 \\
\hline $\begin{array}{l}\text { Bacillus } \\
\text { aryabhattai }\end{array}$ & 01 & VCC.16.SC & Endo_9 & $\begin{array}{l}\text { Messua ferrea } \\
\text { Stem }\end{array}$ & 780 \\
\hline Bacillus niacin & 01 & VCC.16.SA & Endo_20 & $\begin{array}{l}\text { Messua ferrea } \\
\text { Stem }\end{array}$ & 786 \\
\hline \multirow[t]{5}{*}{ Bacillus cereus } & \multirow[t]{5}{*}{20} & VCC.8.SB & Endo_19 & $\begin{array}{ll}\text { Ficus } & \text { religiosa } \\
\text { Stem } & \\
\end{array}$ & 778 \\
\hline & & VCC.22.LF & Endo_45 & $\begin{array}{l}\text { Anthocephalus } \\
\text { kadamba leaf }\end{array}$ & 736 \\
\hline & & VCC.15.LG & Endo_41 & $\begin{array}{l}\text { Terminalia arjuna } \\
\text { leaf }\end{array}$ & 780 \\
\hline & & VCC.25.LF & Endo_80 & $\begin{array}{l}\text { Madhuca indica } \\
\text { leaf }\end{array}$ & 774 \\
\hline & & VCC.25.LE & Endo_75 & Madhuca indica & 762 \\
\hline
\end{tabular}




\begin{tabular}{|c|c|c|c|c|c|}
\hline & & & & leaf & \\
\hline & & VCC.15.LD & Endo_77 & $\begin{array}{l}\text { Terminalia arjuna } \\
\text { leaf }\end{array}$ & 777 \\
\hline & & VCC.27.LD & Endo_74 & $\begin{array}{l}\text { Arthrocarpus } \\
\text { iniegrifolia leaf }\end{array}$ & 775 \\
\hline & & VCC.27.LC & Endo_69 & $\begin{array}{l}\text { Arthrocarpus } \\
\text { iniegrifolia leaf }\end{array}$ & 771 \\
\hline & & VCC.27.LB & Endo_73 & $\begin{array}{l}\text { Arthrocarpus } \\
\text { iniegrifolia leaf }\end{array}$ & 769 \\
\hline & & VCC.14.LC & Endo_76 & $\begin{array}{l}\text { Aegle marmelos } \\
\text { leaf }\end{array}$ & 775 \\
\hline & & VCC.26.LD & Endo_88 & $\begin{array}{l}\text { Calmus roteng } \\
\text { leaf }\end{array}$ & 769 \\
\hline & & VCC.26.LC & Endo_87 & $\begin{array}{l}\text { Calmus roteng } \\
\text { leaf }\end{array}$ & 769 \\
\hline & & VCC.26.LE & Endo_89 & $\begin{array}{l}\text { Calmus roteng } \\
\text { leaf }\end{array}$ & 688 \\
\hline & & VCC.18.L & Endo_84 & $\begin{array}{l}\text { Salmalia } \\
\text { malabarica leaf }\end{array}$ & 776 \\
\hline & & VCC.2.LB & Endo_51 & $\begin{array}{l}\text { Emblica } \\
\text { officinalis leaf }\end{array}$ & 791 \\
\hline & & VCC.24.LD & Endo_54 & $\begin{array}{l}\text { Aegle marmelos } \\
\text { leaf }\end{array}$ & 786 \\
\hline & & VCC.24.LE & Endo_59 & $\begin{array}{l}\text { Azadirachta } \\
\text { indica leaf }\end{array}$ & 780 \\
\hline & & VCC.23.LD & Endo_60 & $\begin{array}{l}\text { Mangifera indica } \\
\text { leaf }\end{array}$ & 770 \\
\hline & & VCC.7.LB & Endo_66 & $\begin{array}{l}\text { Bambusa } \\
\text { arundinacea leaf }\end{array}$ & 703 \\
\hline & & VCC.1a.LB & Endo_72 & $\begin{array}{l}\text { Adhatoda vasica } \\
\text { leaf }\end{array}$ & 783 \\
\hline $\begin{array}{l}\text { Klebsiella } \\
\text { pneumonia }\end{array}$ & 01 & VCC.18.RT & Endo_16 & $\begin{array}{l}\text { Salmalia } \\
\text { malabarica root }\end{array}$ & 648 \\
\hline $\begin{array}{l}\text { Alcaligens } \\
\text { species }\end{array}$ & 04 & VCC.15.LB & Endo_93 & $\begin{array}{l}\text { Terminalia arjuna } \\
\text { leaf }\end{array}$ & 773 \\
\hline & & VCC.25.LB & Endo_94 & $\begin{array}{l}\text { Madhuca indica } \\
\text { leaf }\end{array}$ & 763 \\
\hline & & VCC.24.LA & Endo_96 & $\begin{array}{l}\text { Azadirachta } \\
\text { indica leaf }\end{array}$ & 660 \\
\hline & & VCC.20.LC & Endo_67 & $\begin{array}{l}\text { Calotropi } \\
\text { gigantean } \\
\text { leaf }\end{array}$ & 778 \\
\hline
\end{tabular}




\begin{tabular}{|c|c|c|c|c|c|}
\hline \multirow[t]{2}{*}{$\begin{array}{l}\text { Enterobacter } \\
\text { spp }\end{array}$} & \multirow[t]{2}{*}{02} & VCC.19.S, & Endo_2 & $\begin{array}{l}\text { Veteria indica } \\
\text { Stem, }\end{array}$ & 766 \\
\hline & & VCC.8.RA & Endo_30 & $\begin{array}{l}\text { Ficus religiosa } \\
\text { Stem }\end{array}$ & 677 \\
\hline \multirow{2}{*}{$\begin{array}{l}\text { Arthrobacter } \\
\text { globiformis and } \\
\text { Arthrobacter } \\
\text { protophormiae }\end{array}$} & \multirow[t]{2}{*}{02} & VCC.15.SA & Endo_23 & $\begin{array}{l}\text { Terminalia arjuna } \\
\text { Stem }\end{array}$ & 758 \\
\hline & & VCC.24.LC & Endo_449 & $\begin{array}{l}\text { Azadirachta } \\
\text { indica leaf }\end{array}$ & 751 \\
\hline \multirow{2}{*}{$\begin{array}{l}\text { Kocuria } \\
\text { sediminis and } \\
\text { Kocuria rosea }\end{array}$} & \multirow[t]{2}{*}{02} & VCC.4.SD & Endo_91 & $\begin{array}{l}\text { Eugenia } \\
\text { jambolina Stem, }\end{array}$ & 746 \\
\hline & & VCC.4.RB & Endo_21 & $\begin{array}{l}\text { Eugenia } \\
\text { jambolina } \\
\text { Root }\end{array}$ & 749 \\
\hline \multirow{3}{*}{$\begin{array}{l}\text { Micrococcus } \\
\text { luteus } \\
\text { Microcoocus } \\
\text { lulae and } \\
\text { Micrococcus sp }\end{array}$} & \multirow[t]{3}{*}{03} & VCC.11.SC & Endo_14 & $\begin{array}{ll}\text { Butea frondosa } \\
\text { Root, }\end{array}$ & 745 \\
\hline & & VCC.13.SA & Endo_15 & $\begin{array}{l}\text { Jasminum } \\
\text { auriculatum Stem, }\end{array}$ & 712 \\
\hline & & VCC.13.RB & Endo_31 & $\begin{array}{l}\text { Jasminum } \\
\text { auriculatum } \\
\text { Stem, }\end{array}$ & 724 \\
\hline $\begin{array}{l}\text { Pantoea } \\
\text { dispersa }\end{array}$ & 01 & VCC.15.SB, & Endo_10 & $\begin{array}{l}\text { Terminalia arjuna } \\
\text { Stem, }\end{array}$ & 766 \\
\hline \multirow[t]{2}{*}{$\begin{array}{l}\text { Psychrobacter } \\
\text { spp }\end{array}$} & \multirow[t]{2}{*}{02} & VCC.2.LC, & Endo_56 & $\begin{array}{l}\text { Emblica } \\
\text { officinalis leaf }\end{array}$ & 746 \\
\hline & & VCC.14.LB & Endo_52 & $\begin{array}{l}\text { Aegle } \\
\text { marmelos leaf }\end{array}$ & 768 \\
\hline \multirow[t]{4}{*}{$\begin{array}{l}\text { Ochrobacteriu } \\
\text { m spp }\end{array}$} & \multirow[t]{4}{*}{04} & VCC.2.LA & Endo_46 & $\begin{array}{l}\text { Emblica } \\
\text { officinalis leaf }\end{array}$ & 720 \\
\hline & & VCC.23.LB & Endo_57 & $\begin{array}{l}\text { Mangifera indica } \\
\text { leaf }\end{array}$ & 872 \\
\hline & & VCC.22.LB & Endo_58 & $\begin{array}{l}\text { Anthocephalus } \\
\text { kadamba leaf }\end{array}$ & 704 \\
\hline & & VCC.27.LE & Endo_79 & $\begin{array}{l}\text { Artocarpus } \\
\text { iniegrifolia } \\
\text { leaf }\end{array}$ & 711 \\
\hline $\begin{array}{l}\text { Pseudomnas } \\
\text { spp }\end{array}$ & 01 & VCC.3.RC & Endo_22 & \begin{tabular}{|ll} 
Ficus & racemosa \\
Stem & \\
\end{tabular} & 757 \\
\hline $\begin{array}{l}\text { Staphylococcus } \\
\text { hemolyticus }\end{array}$ & 01 & VCC.CLIED & Endo_92 & \begin{tabular}{|l} 
Strychnos \\
muxvomica
\end{tabular} & 769 \\
\hline \multirow{2}{*}{$\begin{array}{l}\text { No significant } \\
\text { sequence } \\
\text { matching }\end{array}$} & \multirow[t]{2}{*}{17} & VCC.5.R, & Endo_3 & $\begin{array}{l}\text { Acacia catechu } \\
\text { root, }\end{array}$ & \\
\hline & & VCC.15.SC, & Endo_6 & $\begin{array}{l}\text { Terminalia arjuna } \\
\text { Stem, }\end{array}$ & \\
\hline
\end{tabular}




\begin{tabular}{|c|c|c|c|c|c|}
\hline & & VCC.16.R, & Endo_8 & $\begin{array}{l}\text { Messua ferrea } \\
\text { root, }\end{array}$ & \\
\hline & & VCC.13.RA, & Endo_11 & $\begin{array}{l}\text { Jasminum } \\
\text { auriculatum Root, }\end{array}$ & \\
\hline & & VCC.3.RB, & Endo_18 & $\begin{array}{l}\text { Ficus racemosa } \\
\text { root, }\end{array}$ & \\
\hline & & VCC.3.RA, & Endo_29 & $\begin{array}{l}\text { Ficus racemosa } \\
\text { root, }\end{array}$ & \\
\hline & & VCC.11.SB, , & Endo_33 & $\begin{array}{l}\text { Butea frondosa } \\
\text { stem, }\end{array}$ & \\
\hline & & VCC.1a.LA & Endo_38 & $\begin{array}{l}\text { Adhatoda vasica } \\
\text { leaf, }\end{array}$ & \\
\hline & & VCC.25.LI, & Endo_44 & $\begin{array}{l}\text { Madhuca indica } \\
\text { leaf, }\end{array}$ & \\
\hline & & VCC.23.LC, & Endo_55 & $\begin{array}{l}\text { Mangifera indica } \\
\text { leaf, }\end{array}$ & \\
\hline & & VCC.25.L, & Endo_63 & $\begin{array}{l}\text { Madhuca indica } \\
\text { leaf, }\end{array}$ & \\
\hline & & VCC.25.LC, & Endo_65 & $\begin{array}{l}\text { Madhuca indica } \\
\text { leaf, }\end{array}$ & \\
\hline & & VCC.27.LA & Endo_68 & $\begin{array}{l}\text { Artocarpus } \\
\text { iniegrifolia root, }\end{array}$ & \\
\hline & & VCC.20.L, & Endo_78 & $\begin{array}{l}\text { Calotropis } \\
\text { gigantean leaf, }\end{array}$ & \\
\hline & & VCC.13.SB, & Endo_90 & $\begin{array}{l}\text { Jasminum } \\
\text { auriculatum stem, }\end{array}$ & \\
\hline & & VCC.4.SD, & Endo_91 & $\begin{array}{l}\text { Eugenia } \\
\text { jambolina Stem, }\end{array}$ & \\
\hline & & VCC.24.LB & Endo_95 & $\begin{array}{l}\text { Azadirachta } \\
\text { indica } \\
\text { leaf }\end{array}$ & \\
\hline $\begin{array}{l}\text { Unculturable } \\
\text { bacteria }\end{array}$ & 02 & VCC.4.SC, & Endo_17 & $\begin{array}{l}\text { Eugenia } \\
\text { jambolina Stem, }\end{array}$ & 733 \\
\hline & & VCC.14.L & Endo_42 & \begin{tabular}{|l|} 
Aegle \\
marmelos \\
leaf
\end{tabular} & 764 \\
\hline $\begin{array}{l}\text { Terribacillus } \\
\text { saccharophilus }\end{array}$ & 01 & VCC.4.SA & Endo_7 & \begin{tabular}{|l|} 
Eugenia \\
jambolina Stem
\end{tabular} & 726 \\
\hline
\end{tabular}


Table.3 No significant sequence matching

\begin{tabular}{|c|c|c|c|c|}
\hline $\begin{array}{l}\text { Code of } \\
\text { endophytic } \\
\text { bacteria }\end{array}$ & $\begin{array}{l}\text { Name of the } \\
\text { plant and plant } \\
\text { part used }\end{array}$ & Gram's Nature & Capsule & Endospore \\
\hline VCC.5.R & $\begin{array}{l}\text { Acacia catechu } \\
\text { root, }\end{array}$ & $\begin{array}{l}\text { Gram positive } \\
\text { cocci }\end{array}$ & + & - \\
\hline VCC.15.SC & $\begin{array}{l}\text { Terminalia } \\
\text { arjuna } \text { Stem, }\end{array}$ & $\begin{array}{l}\text { Gram positive } \\
\text { cocci }\end{array}$ & - & - \\
\hline VCC.16.R & $\begin{array}{l}\text { Messua ferrea } \\
\text { root, }\end{array}$ & $\begin{array}{l}\text { Gram negative } \\
\text { cocci }\end{array}$ & + & - \\
\hline VCC.13.RA & $\begin{array}{l}\text { Jasminum } \\
\text { auriculatum } \\
\text { Root, }\end{array}$ & $\begin{array}{l}\text { Gram positive } \\
\text { cocci }\end{array}$ & + & - \\
\hline VCC.3.RB & $\begin{array}{ll}\text { Ficus } & \text { racemosa } \\
\text { root, } & \\
\end{array}$ & $\begin{array}{l}\text { Gram positive } \\
\text { cocci }\end{array}$ & - & - \\
\hline VCC.3.RA & $\begin{array}{l}\text { Ficus racemosa } \\
\text { root, }\end{array}$ & $\begin{array}{l}\text { Gram positive } \\
\text { cocci }\end{array}$ & + & - \\
\hline VCC.11.SB & $\begin{array}{l}\text { Butea frondosa } \\
\text { stem, }\end{array}$ & $\begin{array}{l}\text { Gram negative } \\
\text { cocci }\end{array}$ & + & - \\
\hline VCC.1a.LA & $\begin{array}{l}\text { Adhatoda vasica } \\
\text { leaf, }\end{array}$ & $\begin{array}{l}\text { Gram negative } \\
\text { rod }\end{array}$ & + & - \\
\hline VCC.25.LI & $\begin{array}{l}\text { Madhuca indica } \\
\text { leaf, }\end{array}$ & $\begin{array}{l}\text { Gram positive } \\
\text { rod }\end{array}$ & + & - \\
\hline VCC.23.LC & $\begin{array}{l}\text { Mangifera indica } \\
\text { leaf, }\end{array}$ & $\begin{array}{l}\text { Gram positive } \\
\text { rod }\end{array}$ & + & + \\
\hline VCC.25.L & $\begin{array}{l}\text { Madhuca indica } \\
\text { leaf, }\end{array}$ & $\begin{array}{l}\text { Gram negative } \\
\text { rod }\end{array}$ & + & - \\
\hline VCC.25.LC & $\begin{array}{l}\text { Madhuca indica } \\
\text { leaf, }\end{array}$ & $\begin{array}{l}\text { Gram positive } \\
\text { rod }\end{array}$ & + & + \\
\hline VCC.27.LA & $\begin{array}{l}\text { Artocarpus } \\
\text { iniegrifolia root, }\end{array}$ & $\begin{array}{l}\text { Gram negative } \\
\text { rod }\end{array}$ & + & + \\
\hline VCC.20.L & $\begin{array}{l}\text { Calotropis } \\
\text { gigantean leaf, }\end{array}$ & $\begin{array}{l}\text { Gram negative } \\
\text { rod }\end{array}$ & + & + \\
\hline VCC.13.SB & $\begin{array}{l}\text { Jasminum } \\
\text { auriculatum } \\
\text { stem, }\end{array}$ & $\begin{array}{l}\text { Gram positive } \\
\text { cocci }\end{array}$ & + & + \\
\hline VCC.4.SD & $\begin{array}{l}\text { Eugenia } \\
\text { jambolina Stem, }\end{array}$ & $\begin{array}{l}\text { Gram positive } \\
\text { cocci }\end{array}$ & + & + \\
\hline VCC.24.LB & $\begin{array}{l}\text { Azadirachta } \\
\text { indica } \\
\text { leaf }\end{array}$ & $\begin{array}{l}\text { Gram positive } \\
\text { rod }\end{array}$ & + & + \\
\hline
\end{tabular}

(Note: +=Present, _= Absent) 
Fig.1 PCR amplification for 16 s rDNA

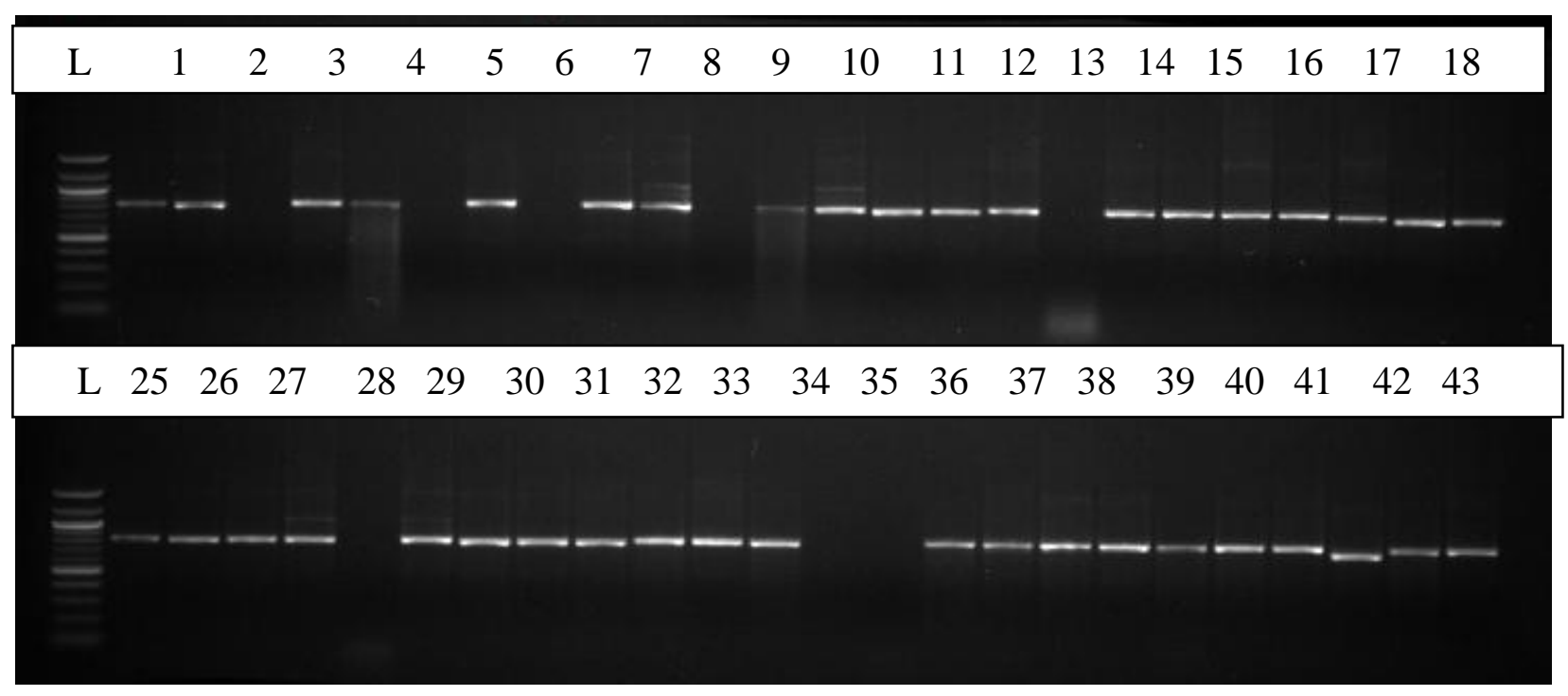

LaneLMarker,lane1VCC.5.S.,lane2:VCC.19.S.,Lane3:VCC.5.R,Lane4:VCC.4.RA,Lane5:11.SA, Lane6:VCC.15.SC,Lane7:VCC.4.SA,Lane8:VCC.16.R,Lane9:VCC.16.SC,Lane10:VCC.15.SB, Lane11:VCC.13.RA,Lane12:VCC.8.SA,Lane13:VCC.3.SA,Lane14:VCC.11SC,Lane15:VCC.13 .SA,Lane16:VCC.18.RT,Lane17:VCC.4.SC,Lane18:VCC.3.RB,Lane19:VCC.8.SB,Lane20:VCC .16.SA,Lane21:VCC.4.SB,Lane22:VCC.3.RC,Lane23:VCC.15.SA,Lane24:VCC.4.SD,Lane25:V CC.9.SB,Lane26:VCC.27.S,Lane27:VCC.27.R,Lane28:VCC.3.SB,Lane29:VCC.3.RA,Lane30:V CC.8.RA,Lane31:VCC.13.RB,Lane32:VCC.4.RB,Lane33:VCC.11.SA,Lane34:VCC.25.LG,Lan e35:VCC.22.LD,Lane36:VCC.15.LF,Lane37:VCC.26.L,Lane38:VCC.1a.LA,Lane39:VCC.25.L H,Lane40:VCC.22.LE,Lane41:VCC.15.LG,Lane42:VCC.14.L,Lane43:VCC.15.L,Lane44:VCC. 25.LI,Lane45:VCC.22.LF,Lane46:VCC.2.LA,Lane47:VCC.23.LA,Lane48:VCC.15.LC.

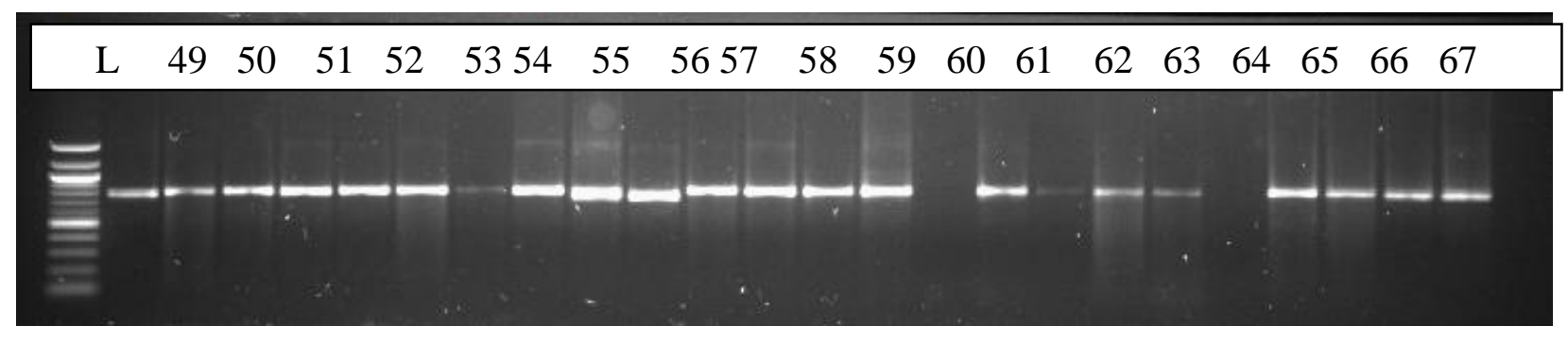

L:Marker,Lane49:VCC.24.LC,Lane50:VCC.22.LG,Lane51:VCC.2.LB,Lane52:VCC.1.4.LB,Lan e53:VCC.22.LA,Lane54:VCC.24.LD,Lane55:VCC.23.LC,Lane56:VCC.2.LC,Lane57:VCC.23.L B,Lane58:VCC.22.LB,Lane59:VCC.24.LE,Lane60:VCC.23.LD,Lane61:VCC.7.LA,Lane62:VC C.23.LB,Lane63:VCC.25.L,Lane64:VCC.12.L,Lane65:VCC.25.LC,Lane66:VCC.7.LB,Lane67: VCC.20.L,Lane68:VCC.27.LC,Lane69:VCC.27.LC,Lane70:VCC.25.LD,Lane71:VCC.7.LC,Lan e72:VCC.1a.LB 


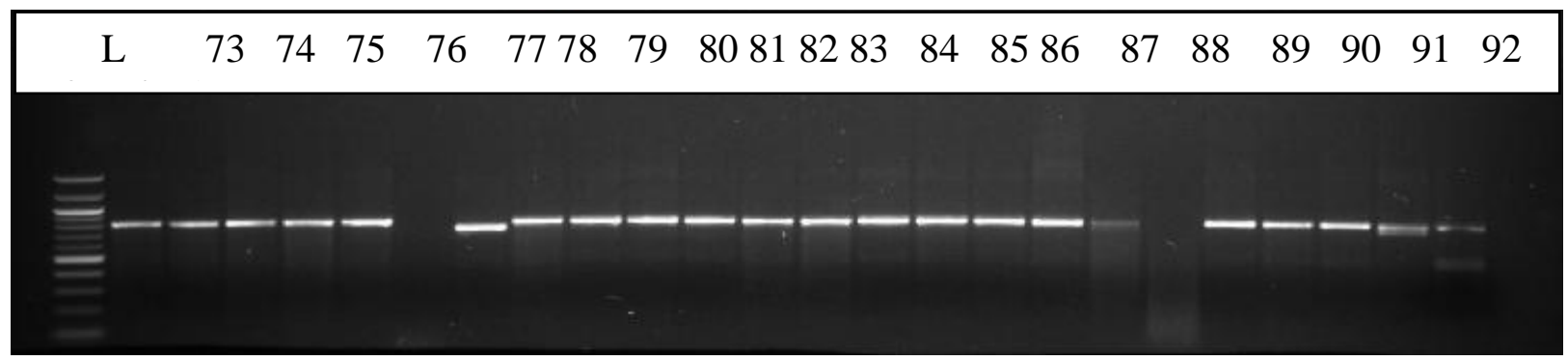

L:Marker,Lane73:VCC.27.LB,Lane74:VCC.27.LD,Lane75:VCC.25.LE,Lane76:VCC.14.LC,La ne77:VCC.15.LD,Lane78:VCC.20.L,Lane79:VCC.27.LE,Lane80:VCC.25.LF,Lane81:VCC.22. LC,Lane82:VCC.15.LE,Lane83:VCC.21.L,Lane84:VCC.18.L,Lane85:VCC.21.LB,Lane86:VCC .26.LB,Lane87:VCC.26.LC,Lane88:VCC.26.LD,Lane89:VCC.26.LE,Lane90:VCC.13.SB,Lane9 1:VCC.4.SD,Lane92:VCC,Lane93:VCC.15.LB,Lane94:VCC.25.LB,Lane95:VCC.24.LB,Lane9 6:VCC.24.LA

Fig.2 Phylogenetic tree analysis

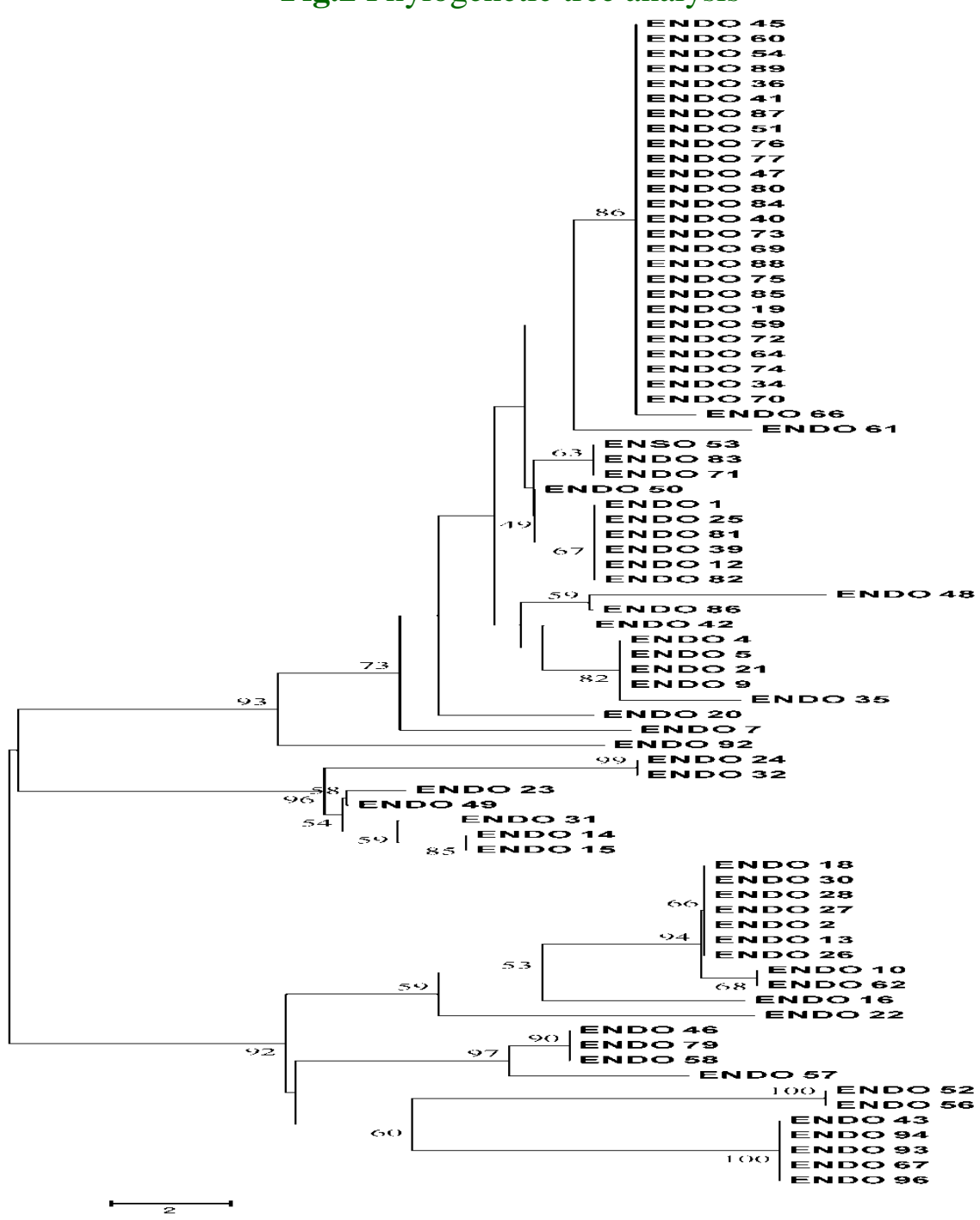


Other genera found to contribute to these humongous tree genomes are Klebsiella pneumoniae, Alcaligens species, Enterobacter spp Arthrobacter globiformis and Arthrobacter protophormiae, Kocuria sediminis and Kocuria rosea, Micrococcus luteus, Microcoocus lulae and Micrococcus sp, Pantoea dispersa, Psychrobacter spp, Ochrobacterium spp, Pseudomnas spp, Staphylococcus hemolyticus, Unculturable bacteria, Terribacillus saccharophilus in tree Salmalia malabarica root, Terminalia arjuna leaf, Madhuca indica leaf, Azadirachta indica leaf, Calotropi gigantean leaf, Veteria indica Stem, Ficus religiosa Stem, Terminalia arjuna Stem, Azadirachta indica leaf, Eugenia jambolina Stem, Eugenia jambolina Root, Butea frondosa Root, Jasminum auriculatum Stem, Jasminum auriculatum Stem, Terminalia arjuna Stem, Emblica officinalis leaf, Aegle marmelos leaf, Emblica officinalis leaf, Mangifera indica leaf, Anthocephalus kadamba leaf, Artocarpus iniegrifolia leaf, Ficus racemosa Stem, Strychnos muxvomica, Eugenia jambolina Stem, Aegle marmelos leaf, Eugenia jambolina Stem. An interesting finding of these endophytic bacterial isolation is as many as 17 isolates are amongst those which could not have significant matching with any of the known sequences on NCBI. Further biochemical investigation to characterize them shall provide a novel appendage to the present culture collection. Bioprospecting through these endophytic bacteria producing useful metabolites would make a valid value addition to the whole process of culture collection.

\section{Acknowledgment}

The authors are very thankful to Department of Biotechnology, New Delhi for financial support and also to VSBT for providing laboratory for experiments.

\section{References}

Andréa Cristina Bogas, et al. 2015. Endophytic bacterial diversity in the phyllosphere of Amazon Paullinia cupana associated with asymptomatic and symptomatic anthracnose. Springer Plus, 4:258.

Anping Peng, et al. 2015. Diversity and distribution of $16 \mathrm{~S}$ rRNA and phenol monooxygenase genes in the rhizosphere and endophytic bacteria isolated from PAH-contaminated sites. Scientific Reports |5: 12173.

Bashan, Y., Holguin, G. 1998. Proposal for the division of plant growth-promoting rhizobacteria into two classifications: biocontrol-PGPB (plant growthpromoting bacteria) and PGPB. Soil Biol. Biochem., 30, 1225e1228.

Chen, C., Bauske, E.M., Musson, G., Rodríguez-Kábana, R. and Kloeppler, J.W. 1995. Biological control of Fusarium wilt on cotton by use of endophytic bacteria. Biol. Control, 5: 83-91.

Ergun, N., S.F. Topcuoglu and A. Yildiz. 2002. Auxin (Indole-3-acetic acid), gibberellic acid $\left(\mathrm{GA}_{3}\right)$, abscisic acid (ABA) and cytokinin (Zeatin) Production by some species of mosses and lichens. Turk. J. Bot., 26: 13-19.

Eu Jin Chung, et al. 2015. Bacillus oryzicola sp. nov., an Endophytic Bacterium Isolated from the Roots of Rice with Antimicrobial, Plant Growth Promoting, and Systemic Resistance Inducing Activities in Rice. Plant Pathol. J., 31(2): 152-164.

Gunter Brader, et al. 2014. Metabolic potential of endophytic bacteria. Curr. Opinion in Biotechnol., 27:30-37.

Gusmaini, Sandra Arifin Aziz, et al. 2013. Isolation and Selection of Endophytic bacteria consortia From Medicinal Plant (Andrographis Paniculata) As 
Plant Growth Promoting Agents. $J$. Agronomy, 12 (3): 113-121.

Hallmann, J., Quadt-Hallmann, A., Mahaffee, W. F., and Kloepper, J. W. (1997). Bacterial endophytes in agricultural crops. Can. J. Microbiol., 43: 895-914.

Huawei Zhang, Chen Ying and Xuelian Bai. 2014. Advancement in Endophytic Microbes From Medicinal Plants. IJPSR, Vol. 5 (5): 1589-1600.

Jasim, B. et al. 2013. Isolation and characterization of plant growth promoting endophytic bacteria from the rhizome of Zingiber officinale. 3 Biotech., 4: 197-204.

Jeyanthi, V., P. Ganesh. 2013. Production, Optimization and Characterization of Phytohormone Indole Acetic Acid by Pseudomonas fluorescence. Int. J. Pharmaceutical \& Biol. Arch., 4(2): $514-520$.

Kartikeya Tiwari and Haresh Kumar Thakur. 2014. Diversity and Molecular Characterization of Dominant Bacillus amyloliquefaciens (JNU-001) Endophytic Bacterial Strains Isolated from Native Neem Varieties of Sanganer Region of Rajasthan. J. Biodivers Biopros. Dev., 1: 115.

Kharwar, R.N., V.C. Verma, G. Strobel and D. Ezra. (2008) The endophytic fungal complex of Catharanthus roseus (L.) G. Don. Curr. Sci., 95: 228-233.

Lalande, R., Bissonett,e N., Coutlée ,D. and Antoun, H. 1989. Identification of rhizobacteria from maize and determination of their plant-growth promoting potential. Plant Soil, 115, 7-11.

Maria Bintang, et al. 2014).Analysis of $16 \mathrm{~S}$ rRNA Sequence of Endophytic Bacteria Isolate. BS1 from Piper betle [L.] Stem. International Conference on Agricultural, Environmental and
Biological Sciences (AEBS-2014), 6970.

Maroof Ahmed, muzaffer Hussain, manoj K. Dhar and Sanjana Kaul. 2012. Isolation of microbial endophytes from some ethnomedicinal plants of Jammu and Kashmir. J. Nat. Prod. Plant Resour., 2(2): 215-220.

Mbai, F.N., et al. 2013. Isolation and Characterisation of Bacterial Root Endophytes with Potential to Enhance Plant Growth from Kenyan Basmati Rice. American Int. J. Contemporary Res., Vol. 3 No. 4:25-40.

Nascimento, S.B., A.M. Lima, B.N. Borges and C.R.B. de Souza. 2015. Endophytic bacteria from Piper tuberculatum Jacq.: isolation, molecular characterization, and in vitro screening for the control of Fusarium solani f. sp piperis, the causal agent of root rot disease in black pepper (Piper nigrum L.) Genetics and Mol. Res., 14(3): 75677577.

Pedraza, R.O., Ramirez-Mata, A., Xiqui, M.1. and Baça Be. 2004. Aromatic amino acid aminotransferase activity and indole-3-acetic acid production by associative nitrogen-fixing bacteria. FEMS Microbiol. Lett., 233: 15-21.

Rhoden, S.A., et al. 2015. Phylogenetic analysis of endophytic bacterial isolates from leaves of the medicinal plant Trichilia elegans A. Juss. (Meliaceae). Genet. Mol. Res., 14(1): 1515-1525.

Shukla, S.T. et al. 2014. Endophytic microbes: A novel source for biologically/pharmacologically active secondary metabolites. Asian $J$. Pharmacol. Toxicol., 02(03): 01-16.

Susheel Kumar, Nutan Kaushik and Peter Proksch. 2013. Identification of antifungal principle in the solvent extract of an endophytic fungus 
Chaetomium globosum from Withania somnifera. Springerplus, 2: 37.

Taghavi, S., Garafola, C., Monchy, S., Newman, L., Hoffman, A., Weyens, N., et al. 2009. Genome survey and characterization of endophytic bacteria exhibiting a beneficial effect on growth and development of poplar trees. Appl. Environ. Microbiol., 75: 748-757.
Vania Specian, Maria Helena Sarragiotto, João Alencar Pamphile and Edmar Clemente. 2012. Chemical characterization of bioactive compounds from the endophytic fungus Diaporthe helianthi isolated from Luehea divaricata. Braz. J. Microbiol., 43(3): 1174-1182.

\section{How to cite this article:}

Priya Dnyandeo Kakade and Sushma Ravindra Chaphalkar. 2016. Phylogenetic Analysis of Endophytic Bacteria from Nakshtra Trees. Int.J.Curr.Microbiol.App.Sci. 5(12): 565-582. doi: http://dx.doi.org/10.20546/ijcmas.2016.512.062 